\title{
Hepatic Abscesses as the Primary Presentation of Crohn's Disease
}

\author{
Dr A Talwar \\ MBBS
}

Senior House Officer

Maj D Edwards

ChM, FRCSEd, RAMC

Specialist Registrar

\section{Mr BJ Moran \\ MCh, FRCSI \\ Consultant Surgeon}

Colorectal Research Unit, North Hampshire Hospital, Aldermaston Road, Basingstoke, Hants RG24 9NA.

SUMMARY: Hepatic Abscesses have been reported in association with Crohn's disease (CD). They either occur as a complication of known CD, or as in this case, are the presentation of undiagnosed disease. The management of the CD associated with liver abscesses is a matter of debate. We report a case of CD presenting with hepatic abscesses, review the literature and discuss the management options.

\section{Introduction}

The most common cause of liver abscess worldwide is amoebiasis. In temperate climates, where amoebiasis is relatively uncommon, liver abscesses present infrequently and are usually pyogenic in origin. The most common causes of pyogenic hepatic abscess are intro-abdominal abscess secondary to appendicitis or diverticulitis and bile-duct infection with ascending cholangitis. A rare cause of hepatic abscess is inflammatory bowel disease.

\section{Case Report}

A 49 year old man was admitted with a 3 week history of fevers, rigors and right-sided abdominal pain. He gave a history of weight loss but no change in bowel habit. Physical examination revealed no abnormality other than right hypochondrial tenderness. A full blood count showed a leucocytosis of $10.4 \times 10^{9}$ cells/L. Serum urea, electrolytes, bilirubin and transaminases were normal; alkaline phosphatase was raised at $135 \mathrm{iU} / \mathrm{L}$. Following ultrasonography of the abdomen, which showed two hypoechoic lesions in the liver, the patient was commenced on parenteral amoxycillin, gentamicin and metronidazole. A CT scan of the abdomen confirmed an abscess (Fig 1) and a diffuse thickening of the

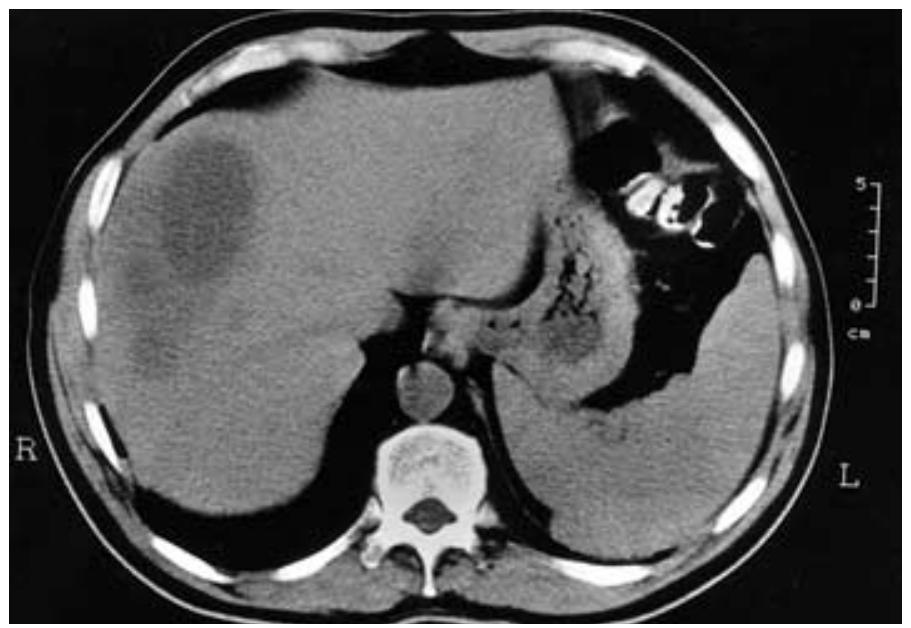

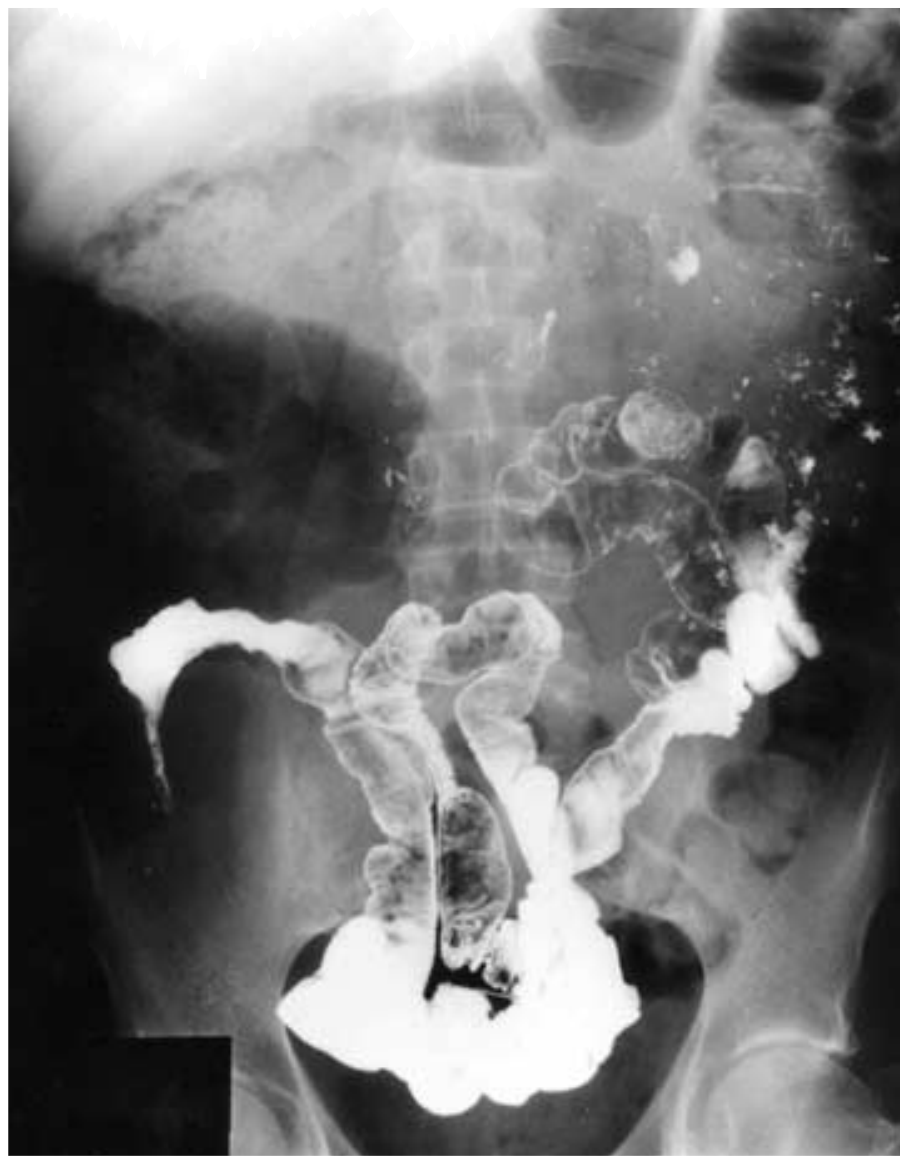

Fig 2.

terminal ileum. Percutaneous drainage of the abscess was performed under ultrasound guidance. Culture of the aspirate yielded microaerophilic Streptococci. A barium follow-through examination of the small intestine revealed narrowing and ulceration of the terminal ileum (Fig 2); the colon was normal at colonoscopy. The patient's symptoms improved and he was sent home on oral antibiotics awaiting a date for terminal ileal resection.

Two weeks later the patient was readmitted with pyrexia and

Fig 1. 
tachycardia. On examination he had peritonism and a mass in the right iliac fossa. At laparotomy a localised abscess, arising from perforated Crohn's terminal ileitis, was found. A limited ileo-caecectomy was performed from which the patient made an uneventful postoperative recovery. He was discharged home after 9 days. Histology of the resected bowel showed actively inflamed small bowel with mucosal ulceration.

\section{Discussion}

Hepatic abscesses presenting in patients with CD are more usually multiple rather than single and have a portal rather than a biliary source of infection. A number of factors are thought to predispose to the formation of abscesses in $\mathrm{CD}$ which include intra-abdominal abscess, fistulas, metronidazole therapy, steroid therapy, surgery and malnutrition. The exact mechanism of seeding of such abscesses is unknown but Greenstein et al proposed a number of possible routes (1). Propagation may occur from a coexisting intra-abdominal abscess, or from the ensuing portal bacteraemia, with or without pylephlebitis. In addition to these portal causes, biliary infection may develop as a result of cholelithiasis, pericholangitis or sclerosing cholangitis, all of which are relatively common biliary complications of inflammatory bowel disease.

There have been over 30 cases reported of hepatic abscess complicating $\mathrm{CD}$, although in only 7 has the patient presented with abscess without a previous diagnosis of CD (2-7). As with our case, all 7 previous reported cases have been male, indeed there is a marked male preponderance in all cases of hepatic abscess associated with CD.

The management of the patient must involve both the management of the abscess and, we believe, the management of the CD. Kotanagi et al used systemic antibiotics to successfully treat a hepatic abscess, confirmed by repeated CT scans and at subsequent laparotomy when the $\mathrm{CD}$ affected transverse colon was resected (3). The majority of authors report aspiration or drainage of the abscess, performed using ultrasound or CT guidance. However, Teague et al indicated that when percutaneous drainage failed to improve the patients clinical condition, formal open drainage may be indicated (they also resected the $\mathrm{CD}$ affected bowel at the same laparotomy (2). Irrespective of the method or not of drainage, hepatic abscesses are treated with systemic antibiotics. The duration of antibiotic therapy remains contentious, but it seems that a period of 4-8 weeks is recommended (8). A further area of controversy is the management of the CD itself. If not suitable for limited or local resection, aggressive medical treatment with amino salicylic acid compounds is indicated. However, the use of steroids in patients with resolving liver abscesses is more contentious. Darnell et al reported the successful treatment of a 38 year old male with pancolitis and terminal ileitis by percutaneous abscess drainage, two weeks of systemic ampicillin and a cephalosporin, followed by a long course of oral steroids (4). Sepsis in CD, and therefore seeding, are thought to be promoted by the use of corticosteroids because of their immunosuppressant effect. However, in a series of 6 patients Vakil et al (5) describe only one patient who was being treated with oral corticosteroids at the time of diagnosis of the hepatic abscess.

Resection of the diseased bowel, after initial presentation with a hepatic abscess, remains controversial. Some reports recommend percutaneous drainage of the abscess with antibiotic therapy $(4,5)$. In this situation the underlying source of sepsis remains and therefore there is a theoretical risk of the complication occurring again, although there have been no case reports in the literature. Other reports recommend that the diseased area of bowel should be resected to prevent further complications. An unusual feature in this case of $\mathrm{CD}$ presenting with hepatic abscess was the subsequent presentation with a second complication of $\mathrm{CD}$, namely perforation whilst awaiting terminal ileal resection. The delay in resection resulted from a desire to allow liver abscess resolution prior to resection of a severely diseased, though relatively symptom free terminal ileum until perforation occurred. We suggest that limited CD presenting as an hepatic abscess should be resected at the earliest opportunity.

\section{References}

1. Greenstein AJ, Sachar DB, Lowenthal D, et al. Pyogenic liver abscess in Crohn's disease. Q J Med 1985; 56(220): 505-18.

2. Teague M, Baddour LM, Wruble LD. Liver Abscess; A harbinger of Crohn's disease. Am J Gastroenterol 1988; 83: 1412-1414.

3. Kotanagi H, Sone S, FukUOKa T, et al. Liver abscess as the initial manifestation of colonic Crohn's disease: Report of a case. Jpn J Surg 1991; 21: 348-351.

4. Darnell A, Brullet E, Campo R, Donoso Li. Liver abscesses as initial presentation of Crohn's disease. Am J Gastroenterol 1995; 90: 1363-1364.

5. Vakil N, Hayne G, Sharma A, Hardy DJ, Slutsky A. Liver abscess in Crohn's disease. Am J Gastroenterol 1994; 89: 1090-1095.

6. Czernichow B, Filippi de la Palavesca MM, Bergier JM, et al. Liver abscess revealing Crohn's disease. Gastroenterol Clin Biol 1993; 17: 153-5.

7. Tung JY, Johnson JL, Liacouras CA. Portal-mesenteric pylephlebitis with hepatic abscesses in a patient with Crohn's disease treated successfully with anticoagulation and antibiotics. J Pediatr Gastroenterol Nutr 1996; 23: 474478.

8. Mir-Madjlessi S, McHenry M, FARMer R. Liver abscess in Crohn's disease. Gastroenterol 1986; 91: 987-93. 